\title{
Newsfronts
}

\section{Second Childhood for Neuronal Precursors}

New research has revealed a gene responsible for locking developing neuronal precursors into a restricted developmental path, indicating the possibility of 'turning back the clock' on neuronal development by targeting the action of specific genes.

The mammalian cerebral cortex consists of six layers, which result from an orderly series of migration and differentiation events during the course of cortical development. There is general recognition that developing neuronal precursors can only produce cells from later stages in the developmental pathway and are blocked from producing cells from earlier stages, although the nature of this restriction has yet to be explained.

The cortex grows in an inside-to-outside manner, with cells migrating outward from the innermost layer to produce successive, more developed layers; consequently, the developmental point in time at which a precursor is generated will determine its ultimate developmental fate. Among the earliest cells to emerge in this process are the Cajal-Retzius (CR) cells, which reside in layer 1 and are recognized as having an essential function in subsequent cortical development events.

Foxg1 is one of the few known genes involved in the earliest stages of cortical development, a transcription factor whose action regulates cell number in the early cortex. Researchers led by Gord Fishell of the New York University Medical Center generated a series of transgenic mice with modified expression of Foxg1 to analyze the role of this gene in cortical development (Science, 2 January).

Mice entirely lacking Foxg1 did not survive past embryonic day 18.5 , but these late-stage embryos showed a striking phenotype: their cortices failed to develop the mature laminar organization seen in wildtype brains, and they appeared to contain excessive quantities of CR cells. Using a Foxg1-driven $L a c Z$ reporter to identify Foxg1-expressing cells in otherwise wildtype mice, Fishell's group determined that Foxg expression is absent in and appears to repress the production of CR cells, and that this gene might be responsible for blocking the generation of early neuronal types over the course of cortical development.

Support for these findings came from additional studies using knockout mice in which Foxg1 expression had been 'rescued' through the inclusion of a conditional expression system. In these mice, the Foxg1 gene would remain active unless exposed to the compound doxycycline, which would transiently repress gene expression and restore the knockout genotype.

Cortical formation was rescued in these mice. However, treatment with doxycycline at embryonic day 13 led to the appearance of new CR cells, even though this is typically the stage at which layer 5 neurons are being produced. Staining with BrdU, a compound that labels only actively dividing cells, confirmed that these CR cells had appeared after the deactivation of the Foxg1 gene.

These findings provide surprising evidence that the capacity of developing neurons to produce earlier cell types is normally blocked but may not be entirely lost. The authors anticipate further investigations to determine exactly how far neu- ronal progenitors can develop before they lose the capacity to have their multipotency restored.

- Michael Eisenstein

\section{A Modern Examination of an Ancient Cure}

A recent study has identified the mode of action, as well as a major active ingredient, for a traditional Chinese herbal remedy for neonatal jaundice, uncovering information that could potentially assist in the identification of new targets and the design of new therapies for this disorder.

Bilirubin is a toxic by-product from the breakdown of hemoglobin, and the action of a series of specialized metabolic enzymes generally clears it from the system. When bilirubin accumulates in the circulation, the yellowish discoloration of the skin known as jaundice results; this disorder is particularly common among newborns and can result in severe developmental damage if not treated promptly.

Yin Zhi Huang, a natural remedy for neonatal jaundice, is a mixture of extracts from four different plants that people throughout Asia have used for hundreds of years. A number of previous studies have confirmed the beneficial effects of Yin Zhi

\section{Cholesterol-Free Mice}

Researchers have created a strain of viable cholesterol-free mice, which may help shed light on the molecule's biological roles in humans. Although excess cholesterol is associated with health problems including heart attacks and strokes, the fatlike molecule has a number of important functions: it is a cell membrane component and a precursor to sex steroids and number of other signaling molecules.

Elena Feinstein of Quark Biotech (Cleveland, $\mathrm{OH}$ ) and her colleagues knocked out the gene Dhcr24, which encodes for an enzyme that converts a cholesterol precursor, desmosterol, to cholesterol. Dhcr $24^{-/-}$pups were $25 \%$ smaller than their Dhcr $24^{+/+}$and Dhcr $24^{+/-}$littermates and were infertile (Science, 19 December 2003). The double knockouts, which have survived to adulthood, accounted for $10-17 \%$ of pups born from $D h c r 24^{+/-}$matings, rather than the expected $25 \%$, suggesting a degree of embryonic lethality.

This phenotype is mild compared with that seen in humans with the extremely rare condition desmosterolosis, which results from mutations in the DHCR24 gene. A possible explanation for this discrepancy is that developing mice can absorb cholesterol from their mothers, while human embryos cannot. These results also suggest that, at least in mice, desmosterol is able to replace cholesterol in a number of functions.

- Tanja Schub 
Huang as a preventative agent and as a treatment for neonatal jaundice, although as yet there is not a clear understanding of the specific compounds responsible for these effects and the mechanisms by which they operate.

To this end, David Moore and his colleagues at the Baylor College of Medicine (Houston, TX) set out to study the mode of action of Yin Zhi Huang, conducting a series of experiments in mice to study more closely the effects of this mixture on bilirubin clearance (J. Clin. Invest., January). After intravenous injection with bilirubin, treatment with Yin Zhi Huang led to a marked increase in the rate of clearance of bilirubin from both the circulatory system and the liver, as well as an increase in the expression of a number of known drug- and xenobiotic-metabolizing enzymes.

Studies have identified constitutive androstane receptor (CAR) NR1I3 as functioning importantly in the process of bilirubin clearance, and Moore's group examined whether Yin Zhi Huang's effects might be mediated by CAR. Knockout mice lacking CAR were largely unresponsive to Yin Zhi Huang treatment, strongly suggesting that CAR plays an important role in this mixture's therapeutic mechanism.

In a series of follow-up experiments, Moore's group used transgenic mice expressing a 'humanized' CAR transgene to identify some of the active ingredients in Yin Zhi Huang. Moore's group identified one of the four herbs, Yin Chin (Artemisia capillaris Miq.), as a major contributor to the mixture's beneficial effects, then went on to identify one of the therapeutically active compounds from Yin Chin, 6,7-dimethylesculetin (DE). As was seen in the mice treated with Yin Zhi Huang, humanized $C A R$ mice treated with either Yin Chin or purified DE exhibited a dose-dependent increase in bilirubin clearance and the expression of drugmetabolizing enzymes.

The results from this study have thus led to the identification of both a therapeutic agent and a target for the treatment of hyperbilirubinemia, and the authors suggest that these findings could be of considerable value in the future development of drugs for curing various forms of jaundice.

$$
-M . E \text {. }
$$

\section{Mouse Model of Pancreatic Cancer}

Scientists have created a long-sought mouse model of pancreatic cancer, possibly opening the door for the development of new screening methods and therapies. Pancreatic cancer is the fifth leading cause of cancer deaths in the United States, killing approximately 30,000 Americans each year, usually within a few months of being diagnosed. In the absence of any techniques for early detection, by the time pancreatic cancers are found, they are usually too far advanced to be removed surgically. In addition, this type of cancer fails to respond to chemotherapy and radiation.

Now, a group led by Ronald A. DePinho of the Dana-Farber Cancer Institute (Boston, MA) report the creation of a mouse strain that develops metastatic pancreatic ductal adenocarcinoma that mimics the condition in humans (Genes Dev., 15 December 2003). The research team found that mice with an activated mutant version of the oncogene Kras and an inactive tumor suppressor gene Ink4a/Arf developed highly invasive pancreatic cancer and died by 11 weeks of age. The presence of both genetic abnormalities was necessary for the development of cancer.

This new mouse model may prove to be a highly effective tool in the understanding of the development of this type of cancer. Furthermore, because these mice develop cancer by the same genetic pathway as do humans, studying them at different time points may lead to the discovery of biomarkers that can be used for early detection of pancreatic cancer in humans.

$-T . S$. 